\title{
Aprendizaje basado en proyectos (ABP) en la formación inicial de maestros en la Escuela Normal Superior de Salamina, Caldas, Colombia
}

Gustavo Andrés Cortés Gallego*

Recibido: 04-08-2019

Aceptado: 06-05-2020

Citar como: Cortés Gallego, G. A. (2021). Aprendizaje basado en proyectos en la formación inicial de maestros en la Escuela Normal Superior de Salamina, Caldas, Colombia. Revista Interamericana de Investigación, Educación y Pedagogía, 14(2), 299-315. https://doi. org/10.15332/25005421.6640

\section{Resumen}

La presente propuesta de investigación busca analizar una experiencia de aprendizaje basado en proyectos (ABP) para la formación inicial de maestros en la Escuela Normal Superior de Salamina, Caldas, Colombia. En tal sentido, una comprensión general se construye desde las voces de los sujetos que viven la experiencia. Así, la metodología elegida es la cualitativa que, desde la propuesta de la teoría fundada de Strauus y Glasser (2016), permitirá no solo reconstruir la experiencia, sino también reconocer el sentido y significado que los sujetos le dan a esta. Así las cosas, los objetivos específicos están relacionados con las etapas fundamentales del proceso de construcción de conocimiento propuestas por los autores mencionados. El primer objetivo específico busca identificar, de manera emergente, las categorías que dan sentido

\footnotetext{
* Ingeniero químico por la Universidad Nacional de Colombia, sede Manizales; magíster en Educación por la Universidad Santo Tomás, sede Bogotá. Docente del Programa de Formación Complementaria de la Escuela Normal Superior de Salamina, Caldas, Colombia.

Correo electrónico: cortesga@hotmail.com

ORCID: https://orcid.org/0000-0003-3611-378X
} 
a la experiencia del $A B P$; el segundo objetivo pretende reconocer las relaciones entre dichas categorías; y, el tercero, establecer la síntesis teórica de dicha experiencia. Para todo esto los registros de diarios de campo y grupos focales serán los instrumentos privilegiados. Esta propuesta se diferencia de trabajos encontrados que, desde posturas cuantitativas, buscan evaluar deductivamente las teorías relacionadas con el ABP, como trabajo cooperativo, actitud de estudiantes, desarrollo de competencias, entre otros aspectos.

Palabras clave: aprendizaje basado en proyectos (ABP), formación de maestros, metodología cualitativa, teoría fundamentada.

\section{Project-Based Learning (PBL) in Initial Teacher Training at Escuela Normal Superior de Salamina, in Caldas, Colombia}

\section{Abstract}

This research proposal seeks to analyze a project-based learning $(\mathrm{PBL})$ experience for initial teacher training at Escuela Normal Superior de Salamina, in Caldas, Colombia. In this sense, a general understanding is built from the voices of the subjects who live the experience. Thus, the chosen methodology is qualitative, based on the proposal of the grounded theory of Strauss and Glasser (2016), which will allow us not only to reconstruct the experience but also to recognize the sense and meaning that the subjects give to it. The specific objectives are related to the fundamental stages of the knowledge construction process proposed by the aforementioned authors. The first specific objective seeks to identify, in an emergent manner, the categories that give meaning to the $\mathrm{PBL}$ experience; the second objective seeks to recognize the relationships between these categories; and the third, to establish the theoretical synthesis of this experience. To accomplish this, field diary records and focus groups will be the privileged instruments. This proposal differs from other works found that, based on quantitative approaches, seek to evaluate deductively the theories related to 
PBL such as cooperative work, students' attitude, development of competencies, among other aspects.

Keywords: project-based learning, teacher education, qualitative methodology, grounded theory.

\section{Aprendizagem baseada em projetos ( $A B P$ ) para a formação inicial de professores na "Escuela Normal Superior de Salamina" em Caldas, Colômbia}

\section{Resumo}

Esta proposta de pesquisa procura analisar uma experiência de aprendizagem baseada em projetos (ABP) para a formação inicial de professores na "Escuela Normal Superior de Salamina" em Caldas, Colômbia. Nesse sentido, um entendimento completo é construído a partir das vozes dos sujeitos que vivem a experiência. Assim, a metodologia escolhida é qualitativa, o que a partir da proposta da teoria fundamentada de Strauus e Glasser (2016) permitirá não só reconstruir a experiência, mas também reconhecer o sentido e o significado que os sujeitos lhe dão. Assim, os objetivos específicos estão relacionados com as etapas fundamentais do processo de construção do conhecimento proposto pelos autores acima mencionados. O primeiro objetivo específico procura identificar, de maneira emergente, as categorias que dão sentido à experiência $A B P$; o segundo objetivo procura reconhecer as relações entre essas categorias; e o terceiro, estabelecer a síntese teórica dessa experiência. Por tudo isso, os registros diários de campo e os grupos de foco serão os instrumentos privilegiados. Esta proposta difere dos trabalhos encontrados que, a partir de posturas quantitativas, procuram avaliar dedutivamente as teorias relacionadas à $A B P$, tais como trabalho cooperativo, atitude dos estudantes, desenvolvimento de competências, entre outros aspectos.

Palavras-chave: aprendizagem baseada em projetos (ABP), treinamento de professores, metodologia qualitativa, teoria fundamentada. 


\section{Explicación del problema}

La presente propuesta de investigación pretende dar respuesta a la siguiente pregunta de investigación: ¿cómo es una experiencia de aprendizaje basado en proyectos en la formación inicial de maestros en la Escuela Normal Superior María Escolástica de Salamina, Caldas, Colombia? En tal sentido, se plantea como objetivo analizar en dicha institución una experiencia de ese tipo. Con esta intención y al hacer una revisión documental (Kilpatrick, 1929; Pecore, 2015; Thomas, 2000; Moursund, 1999; Blumenfeld et ál., 2011; Barron et ál., 2011; Jurado, 2014; Segura, 2018; Trujillo, 2012) se reconoce la necesidad de comprender, desde una perspectiva cualitativa, las experiencias de los maestros en formación y su ejercicio en una vivencia del ABP. Es así como, desde la metodología de la teoría fundamentada de Strauss y Corbin (2016), se identifican los siguientes ejes fundamentales que, con las preguntas específicas, orientan los objetivos específicos.

\section{Ejes fundamentales}

A partir de la pregunta de investigación, se plantean las siguientes preguntas específicas que orientan los objetivos de investigación y dan cuenta del diseño metodológico asumido:

- Pregunta específica 1: ¿cuáles son los elementos significativos de una experiencia de aprendizaje basado en proyectos en la formación inicial de maestros?

- Objetivo específico 1: identificar los elementos significativos de una experiencia de aprendizaje basado en proyectos en la formación inicial de maestros.

- Pregunta específica 2: ¿cuáles son las relaciones entre los elementos significativos de una experiencia de aprendizaje basado en proyectos en la formación inicial de maestros. 
- Objetivo específico 2: reconocer las relaciones entre los elementos significativos de una experiencia de aprendizaje basado en proyectos en la formación inicial de maestros.

Así las cosas, se presentan a continuación la justificación del proyecto, el marco teórico, el marco metodológico y el calendario de actividades.

\section{Justificación}

En el proceso de revisión preliminar de antecedentes para este proyecto, se encontró la preeminencia de investigaciones de tipo cuantitativo. Estas ponen a prueba - de manera deductiva y por lo general en la educación básica-comprensiones particulares sobre el aprendizaje basado en proyectos (ABP). Se preocupan por aspectos diferentes como la actitud de los estudiantes, el trabajo cooperativo, el desarrollo de competencias, entre otros.

Así, Thomas (2000) plantea tendencias actuales en el proceso de investigación de los procesos de aprendizaje basado en proyectos y reconoce los siguientes intereses investigativos: evaluación sumativa, evaluación formativa, papel de los estudiantes e investigación intervención (p. 8). De igual manera, identifica técnicas como las pruebas estandarizadas, encuestas, evaluación de tareas, observación de clase, entrevista a maestros y análisis de reportes de profesores. Asimismo, presenta algunos hallazgos como son la mejora en el clima escolar, motivación, disminución de ausentismo, entre otros.

En este marco, la presente propuesta busca conocer cómo es la experiencia del aprendizaje basado en proyectos. Esto permitirá fundamentar el proceso de ABP desde las voces de los sujetos en el contexto de la formación inicial de maestros en la Escuela Normal Superior de Salamina, Caldas, Colombia. Por otro lado, en el proceso se logrará la cualificación de maestros, la cual es una tarea 
fundamental de las escuelas normales, que son las encargadas de la formación de los normalistas superiores, maestros encargados, junto con los licenciados de educación preescolar y básica primaria.

\section{Marco teórico}

La propuesta de investigación tiene sus fundamentos en la postura pedagógica que da sentido al aprendizaje basado en proyectos. Es así como se reconocen, en las ideas de Jhon Dewey, las base filosóficas y pedagógicas que sustentan la necesidad de un proceso de formación particular de los sujetos. En tal sentido, Dewey propone la necesidad de:

- Que el niño encuentre por sí mismo situaciones problemáticas en su experiencia.

- Facilitarle la delimitación e intelectualización de la situación problemática.

- Darle oportunidad para que haga sus observaciones y experiencias.

- Reelaboración de las hipótesis para que el niño se forme sus propias ideas y no dárselas estereotipadas de antemano.

- Aplicación de las ideas elaboradas (Rincón, 2012, p. 12).

A partir de estos postulados, William Kilpatrick (1929) propone el Método de proyectos, el cual también fue influenciado por la psicología del aprendizaje de Edward L. Thorndike. (Pecore, 2015, p. 2). De acuerdo con Rincón (2012), Kilpatrick definía los proyectos como:

[...] una actividad preconcebida en el que el designio dominante fija el fin de la acción, guía su proceso y proporciona 
su motivación. Ya en esta definición son notorias las características básicas de un proyecto: planeación previa (preconcebida) de las actividades, coherencia entre objetivos, plan de acción y evaluación, así como motivación intrínseca, originada en el interés que hace surgir el proyecto mismo. (p. 12)

Además, Kilpatrick (1929) planteó cuatro tipos de proyectos: 1) de producción; 2) de consumo, en el cual se aprende a utilizar algo ya producido; 3) de resolución de algún problema; o 4) de perfeccionamiento de alguna técnica.

Dentro de las características del proyecto también estableció:

- "un plan de trabajo de preferencia manual;

- una actividad motivada por medio de una intervención lógica;

- un trabajo manual, teniendo en cuenta la diversidad globalizada de enseñanza; y

- un ambiente natural." (Gadotti, 2003, p. 150)

Recientemente, el ABP ha incorporado diversos desarrollos teóricos. Los trabajos de Jean Piaget, Lev Vygotsky y Jerome Bruner tienen en cuenta los principios del constructivismo (Pecore, 2015, p. 5). Otros desarrollos están relacionados con el uso de la tecnología, el aprendizaje cooperativo, el desarrollo del pensamiento, la evaluación formativa entre otros. Estas concepciones se presentan a continuación.

Es así como en la literatura se encuentra una gran variedad de concepciones sobre el aprendizaje basado en proyectos. Estas van desde la de Thomas (2000, p. 3), para quien el ABP es un conjunto de tareas basadas en la resolución de preguntas o problemas a través de la implicación del alumno en procesos de investigación de manera relativamente autónoma, que culmina con un producto final presentado ante los demás. 
Por su parte, Moursund (1999, p. 12), desde los procesos ABP incorporados en la tecnología, promueve, entre otros aspectos, el desarrollo de procesos de aprendizaje auténtico, la mejora de la motivación, la promoción de las habilidades para la solución de problemas y el uso de la tecnología.

Por otro lado, Blumenfeld et ál. (2011), plantean que el ABP es:

[...] una perspectiva integral centrada en la enseñanza mediante la implicación de estudiantes en procesos de investigación. Dentro de este marco, los estudiantes buscan soluciones a problemas no triviales, formulando y refinando preguntas, debatiendo ideas, haciendo predicciones, diseñando planes y/o experimentos, recopilando y analizando datos, sacando conclusiones, comunicando sus ideas y hallazgos a otros, haciendo nuevas preguntas y creando artefactos. (p. 371)

Para el mismo autor

[...] hay dos componentes esenciales de los proyectos: requieren una pregunta o problema que sirve para organizar e impulsar las actividades; y estas actividades resultan en una serie de artefactos, o productos, que culminan en un producto final que aborda la cuestión de la pregunta inicial. (p. 371)

Lo anterior demuestra la variedad de actividades que implica un proceso ABP y, de otro lado, el sentido que dichas actividades tienen al orientar la búsqueda de la(s) respuesta(s) a la pregunta guía y a la construcción y presentación del producto final.

Entre tanto, Barron et ál. (2011) plantean cuatro principios de los procesos de ABP así: "Objetivos claros, apoyo a estudiantes y maestros, autoevaluación permanente y apoyo institucional" (p. 273). Dichos principios implican, como se ve, el compromiso de la organización escolar. 
Es de destacar que para Jurado (2014) el ABP en un proceso en el que "las interacciones están mediadas por un problema o un centro de interés que empuja hacia la conjetura, es decir, hacia hipótesis interpretativas, que presuponen actitudes hacia la indagación y la pregunta, que desemboca en múltiples preguntas" (p. 20). Así las cosas, el eje fundamental lo constituyen los procesos de investigación.

Más aún, Segura (2018, p. 58) reconoce en el ABP la posibilidad de aprender a "trabajar en equipo y a valorar los aportes de todos" en torno al conocimiento del propio contexto, ya que en el ABP "su origen es lo cotidiano y las dificultades locales, que son la fuente de las búsquedas y actividades".

Finalmente, Trujillo (2012) propone algunos elementos para el ABP como el trabajo en equipo del profesorado, trabajo del alumnado en grupos cooperativos, uso intensivo de las tecnologías de la información y la comunicación (TIC), fomento de la socialización rica y mecanismos alternativos de evaluación. Todo lo anterior le ha dado al aprendizaje basado en proyectos un mayor nivel de complejidad, tanto para su implementación como para su conceptualización.

\section{Marco metodológico}

La investigación pretende analizar una experiencia de aprendizaje basado en proyectos en la formación inicial de maestros en una escuela normal superior. En tal sentido, son las voces de los sujetos que viven la experiencia las que permitirán no solo reconstruirla, sino también reconocer el sentido y significado que los mismos sujetos le atribuyen.

De acuerdo con lo anterior, se reconoce la necesidad de una postura metodológica de corte cualitativo que permita, de manera inductiva, dar cuenta de la experiencia vivida en el desarrollo del proyecto $A B P$. Es este sentido, nos ubicamos en la postura de la 
sistematización de experiencias entendida para Jara (1994, citado en Verger, 2007) como:

[...] el proceso de reconstrucción y reflexión analítica sobre una experiencia de acción o de intervención mediante la cual interpretarla y comprenderla. Con el proceso de sistematización se obtiene un conocimiento consistente que permite transmitir la experiencia, confrontarla con otras experiencias o con el conocimiento teórico existente. Así, se contribuye a la acumulación de conocimientos generados desde y para la práctica, y a su difusión o transmisión. (p. 628)

En este marco, el proceso de sistematización permitirá, a partir del análisis de la experiencia ABP, su comprensión, transmisión, comparación y transformación.

Así las cosas, se plantea inicialmente la revisión de la literatura sobre el aprendizaje basado en proyectos en la formación inicial de maestros en las escuelas normales superiores a nivel nacional e internacional. Se procederá así: búsqueda de la información, organización de los hallazgos, análisis de los datos y conclusiones.

Una vez se lleva a cabo la revisión bibliográfica, se realiza — junto con los estudiantes del curso de investigación educativa- el diseño del proyecto de ABP. Este tendrá una duración de 20 semanas. Luego de iniciado el proyecto, de manera simultánea se realizará el proceso de análisis cualitativo de la experiencia siguiendo los planteamientos de Glasser y Strauss, donde se propone un abordaje inductivo del proceso de construcción de conocimiento en etapas de: 1) recolección de datos; 2) codificación abierta; 3) codificación axial; 4) codificación selectiva; y 5) visualización de la teoría (Hernández, 2010, p. 492).

El cumplimiento de los objetivos específicos se irá dando forma de la siguiente manera: 
Objetivo específico 1. Identificar los elementos significativos de una experiencia de aprendizaje basado en proyectos en la formación inicial de maestros

Durante el desarrollo del proyecto ABP se realiza el proceso de recolección de datos. Se recolecta información por medio de los diarios de campo del maestro en ejercicio y los maestros en formación (estudiantes). De igual modo, se realizan grupos focales cada 15 días para hacer el seguimiento colectivo de la experiencia; estos grupos focales son registrados en actas. A medida que se van generando los registros de diarios de campo y los grupos focales, se realiza el proceso de codificación abierta de la información, etapa en donde:

[...] el investigador revisa todos los segmentos del material para analizar y generar por comparación constante categorías iniciales de significado. Elimina así la redundancia y desarrolla evidencia para las categorías (sube de nivel de abstracción). Las categorías se basan en los datos recolectados (entrevistas, observaciones, anotaciones y demás datos). Las categorías tienen propiedades representadas por subcategorías, las cuales son codicadas (las subcategorías proveen detalles de cada categoría). (Hernández, 2010, p. 492)

Como producto de esta etapa se obtienen las categorías iniciales de significado. Estas dan cuenta de los elementos significativos de la experiencia a los que hace referencia el primer objetivo específico.

\section{Objetivo específico 2. Reconocer las relaciones entre los elementos significativos de una experiencia de aprendizaje basado en proyectos en la formación inicial de maestros}

Una vez identificadas las categorías que dan cuenta de la experiencia, se continua con el proceso de codificación axial. Este busca proponer relaciones preliminares entre las categorías para dar 
un sentido global inicial a la experiencia $A B P$, y se procede de la siguiente manera:

De todas las categorías codificadas de manera abierta, el investigador selecciona la que considera más importante y la posiciona en el centro del proceso que se encuentra en exploración (se le denomina categoría central o fenómeno clave). Posteriormente, relaciona a la categoría central con otras categorías. Estas pueden tener distintas funciones en el proceso. (Hernández, 2010, p. 492)

Una vez propuesto este sistema de relaciones, se realiza el proceso de codificación selectiva, en donde se busca evidencias en los registros que den sustento a las relaciones propuestas, así:

Una vez generado el esquema, el investigador regresa a las unidades o segmentos y los compara con su esquema emergente para fundamentarlo. De esta comparación también surgen hipótesis (propuestas teóricas) que establecen las relaciones entre categorías o temas. Así, se obtiene el sentido de entendimiento. (Hernández, 2010, p. 492)

Es decir, en esta etapa lo que se busca es encontrar en los registros la evidencia de las relaciones que se habían propuesto entre las categorías.

Objetivo específico 3. Sistematizar una experiencia de aprendizaje basado en proyectos en la formación inicial de maestros

Aquí se recurre a diversas estrategias para concretar una comprensión particular de la experiencia desde las voces de quienes la viven - visualizar la teoría emergente-. Esto implica regresar permanentemente a los sujetos para socializar y contrastar los hallazgos, los cuales se comparan, además, con los establecidos en la revisión bibliográfica. Es así como "se escribe una historia o narración que vincule las categorías y describa el proceso o fenómeno. 
Se pueden utilizar las típicas herramientas de análisis cualitativo (mapas, matrices, etcétera)." (Hernández, 2010, p. 492).

El final del proceso de recolección y análisis de la información se da cuando ocurre la saturación de categorías; es decir, cuando los datos se vuelven repetitivos o redundantes, y los nuevos análisis confirman lo que se ha fundamentado (Hernández, 2010, p. 492).

\section{Aportación a la ciencia y a la sociedad}

La presente propuesta de investigación permitirá —además de analizar una experiencia de ABP en la formación inicial de maestros, como se describe en la justificación-cualificar los procesos de la escuela normal, teniendo en cuenta su naturaleza y responsabilidades. Es así como en los años recientes la Asociación Nacional de Escuelas Normales de Colombia (Asonen) y el Ministerio de Educación Nacional de Colombia vienen desarrollando trabajos conjuntos para la redefinición del sentido de las escuelas normales; $y$, en tal sentido, en el año 2015 construyeron el documento Naturaleza y retos de las Escuelas Normales Superiores. En dicho documento se establece una concepción para estas instituciones, como se presenta a continuación:

[...] deben entenderse como una institución que es "Centro de Formación Docente" en el que se estudia y produce saber pedagógico, y que se especializa en la formación de docentes para la educación preescolar y básica primaria; es decir la ENS es un escenario donde confluyen, de manera articulada, la formación pedagógica, la investigación, la evaluación educativa y la proyección social, en relación con la educación preescolar y básica primaria, que es el campo de acción de sus egresados. (Ministerio de Educación Nacional de Colombia [MEN], 2015, p. 24)

De lo anterior se reconocen los elementos fundamentales de una escuela normal superior en Colombia, a saber: la formación 
pedagógica, la investigación, la evaluación educativa y la proyección social. En relación con el proceso investigativo de los maestros en formación, este debe desarrollarse en tres ámbitos:

(i) [...] definición de la disposición de indagación y la actitud reflexiva del educador en torno a su praxis, con un sentido investigativo, para avanzar desde la elaboración de estructuras conceptuales derivadas de la experiencia hacia la construcción y afianzamiento del conocimiento; (ii) [...] formación del educador como intelectual y productor de conocimiento académico, con claros avances y apropiación teórica y práctica para la consolidación de la pedagogía como disciplina; y (iii) [...] reconocimiento de la investigación como una alternativa didáctica para el desarrollo de la práctica pedagógica. (MEN, 2015, p. 36)

Los elementos anteriores se constituyen en requerimientos de la formación inicial de maestros que desde el ABP se les puede dar respuesta. Es así como un proyecto ABP en torno a los procesos de práctica pedagógica de los maestros en formación permitiría la construcción de saber pedagógico, la apropiación de la teoría pedagógica y la promoción de la investigación desarrollada desde el ABP como opción didáctica.

\section{Calendario}

En la tabla 1 se presenta el calendario de actividades con cada una de las fases del desarrollo del proyecto de investigación.

Tabla 1. Calendario de actividades.

\begin{tabular}{|l|l|l|l|}
\hline Objetivo & Actividad & Tiempo & Recursos \\
\hline $\begin{array}{l}\text { Objetivo específico 1 } \\
\text { Identificar los elementos } \\
\text { significativos de una } \\
\text { experiencia de aprendizaje } \\
\text { basado en proyectos en la } \\
\text { formación inicial de maestros }\end{array}$ & $\begin{array}{l}\text { Revisión de la literatura. } \\
\text { ABP en la formación inicial } \\
\text { no maestros en las escuelas }\end{array}$ & 2 meses & $\begin{array}{l}\text { Los recursos } \\
\text { institucionales de la } \\
\text { escuela normal. }\end{array}$ \\
\hline & $\begin{array}{l}\text { Diseño del proyecto ABP en } \\
\text { conjunto con los estudiantes }\end{array}$ & 1 mes & Atlasti \\
\hline
\end{tabular}




\begin{tabular}{|c|c|c|c|}
\hline Objetivo & Actividad & Tiempo & Recursos \\
\hline & $\begin{array}{l}\text { Ejecución del proyecto ABP } \\
\text { y recolección de los datos* }\end{array}$ & 4 meses & \\
\hline & $\begin{array}{l}\text { Ejecución del proyecto ABP } \\
\text { y codificación abierta* }\end{array}$ & 4 meses & \\
\hline \multirow{2}{*}{$\begin{array}{l}\text { Objetivo específico } 2 \\
\text { Reconocer las relaciones } \\
\text { entre los elementos } \\
\text { significativos de una } \\
\text { experiencia de aprendizaje } \\
\text { basado en proyectos en la } \\
\text { formación inicial de maestros }\end{array}$} & $\begin{array}{l}\text { Ejecución del proyecto ABP } \\
\text { y codificación axial* }\end{array}$ & 4 meses & \\
\hline & $\begin{array}{l}\text { Ejecución proyecto ABP y } \\
\text { codificación selectiva* }\end{array}$ & 4 meses & \\
\hline \multirow{2}{*}{$\begin{array}{l}\text { Objetivo específico } 3 \\
\text { Formular una comprensión } \\
\text { particular de una experiencia } \\
\text { de aprendizaje basado en } \\
\text { proyectos en la formación } \\
\text { inicial de maestros. }\end{array}$} & $\begin{array}{l}\text { Visualización y } \\
\text { contrastación de la teoría } \\
\text { emergente }\end{array}$ & 2 meses & \\
\hline & Redacción del informe final & 3 meses & \\
\hline
\end{tabular}

* Etapas simultáneas.

Fuente: elaboración propia.

\section{Referencias}

Barron, B. J., Schwartz, D. L., Vye, N. J., Moore, A., Petrosino, A., Zech, L. y Bransford, J. D. (1998). Doing with understanding: Lessons from research on problem-and project-based learning. Journal of the Learning Sciences, 7(3-4), 271-311.

Blumenfeld, P. C., Soloway, E., Marx, R.W., Krajcik, J. S., Guzdial, M. y Palincsar, A. (1991). Motivating project-based learning: Sustaining the doing, supporting the learning. Educational psychologist, 26(3-4), 369398.

Dewey. (1998). Democracia y educación. Morata.

Gadotti, M. (2013). Historia de las ideas pedagógicas (4. ${ }^{\mathrm{a}}$ ed.). Siglo veintiuno editores.

Hernández, R. (2010). Metodología de la investigación. McGrawHill. 
Jara, O. (1994). Para sistematizar experiencias. Alforja.

Jurado, F. (2014). La pedagogía por proyectos vs. la pedagogía según programas estandarizados. Ruta Maestra (9.a edición), 18-24. https:// rutamaestra.santillana.com.co/la-pedagogia-por-proyectos-vs-la-pedagogia-segun-programas-estandarizados/

Kilpatrick, W. H. (1929). The Project Method. The use of the purposeful act in the educative process. Teachers college, Columbia University. http://books.google.com/books?id=4DJCAAAAIAAJ

Ministerio de Educación Nacional de Colombia (MEN). (2012). Sistema colombiano de formación de educadores. https://cms.mineducacion. gov.co/static/cache/binaries/articles-345485_anexo1.pdf?binary_ rand $=4264$

Ministerio de Educación Nacional de Colombia (MEN). (2015). Naturaleza y retos de las Escuelas Normales Superiores. https://www.mineducacion. gov.co/1759/articles-345485_recurso_1.pdf

Moursund, D. G. (1999). Project-based learning using information technology. International society for technology in education.

Pecore, J. L. (2015). From Kilpatrick's project method to project-based learning. International Handbook of Progressive Education, 155-171.

Rincón, G. (2012). Los proyectos de aula y la enseñanza y el aprendizaje del lenguaje escrito. Colombia: Red Colombiana para la Transformación de la Formación Docente en Lenguaje. http://www.lenguaje.red/ docs/2020/Los_proyectos_de_aula_y_ensenanza.pdf

Segura, D. (2018). El trabajo por proyectos es una promesa para la escuela en un país que sueña. Revista Rutas de formación: prácticas y experiencias, 5, 55-59. https://doi.org/10.24236/24631388.n5.2017. 1307

Strauss, A y Corbin, J. (2016). Bases de la investigación cualitativa. Técnica y procedimientos para desarrollar la teoría fundamentada. Universidad de Antioquia. 
Thomas, J. W. (2000). A review of research on project-based learning. https://www.researchgate.net/publication/238162544_A_Review_of_ Research_on_Project-Based_Learning

Trujillo, F. (2012). Enseñanza basada en proyectos: una propuesta eficaz para el aprendizaje y el desarrollo de las competencias básicas. Revista Eufonía-Didáctica de la Educación Musical, 55, 7-15.

Verger, A. (2007). Sistematizando experiencias: análisis y recreación de la acción colectiva desde la educación popular. Revista de Educación, 343, 623-645. 